\title{
Le conflit entre l'ethos préalable et l'ethos discursif dans le discours politique de Sadate prononcé à la Knesset israélienne ${ }^{1}$
}

\author{
MOHAMED SAAD ALI
}

Université du Fayoum, Egypte

hma_saad@yahoo.com

"Parler, c'est sans doute échanger des informations; mais c'est aussi effectuer un acte, régi par des règles précises, qui prétend transformer la situation du récepteur et modifier son système de croyance et/ou son attitude comportementale. "(Kerbrat Orecchioni, 1980, p.84).

\section{Introduction}

Depuis Aristote, il est bien connu que les arguments que présente un orateur dans un discours appartiennent à trois preuves qui recouvrent l'intégralité du travail de persuasion. C'est la triade du logos / pathos / ethos. De ces trois éléments, l'ethos est, pour Aristote, la plus efficace des preuves parce que c'est la crédibilité de l'orateur qui rend le discours plus efficace. En effet, grâce aux récents travaux linguistiques sur l'énonciation, l'ethos connaît un grand essor et devient un élément essentiel de l'analyse rhétorique des discours². Soulignons que c'est surtout Dominique Maingueneau ${ }^{3}$ qui a relancé le travail sur l'ethos en analyse du discours. En plus, des linguistes contemporains vont plus loin en confirmant l'effacement progressif du logos, comme arguments logiques basés sur la raison, au profit de l'ethos et de l'appel au sentiment ${ }^{4}$.

Dans l'étude présente, il s'agit d'un discours politique prononcé par le président égyptien Anouar Al-Sadate à la Knesset israélienne en 1977. Dans ce discours, la crédibilité de l'orateur est mise à mal avant même la prise de parole, c'est-à-dire à cause de son ethos préalable. En analyse du discours, cette représentation antérieure à la prise de parole se trouve « au fondement de l'image qu'il [le locuteur] construit dans son discours : il tente en effet de la consolider, de la rectifier, de la retravailler ou de la gommer ${ }^{5} \gg$. Nous voudrions ainsi retracer les stratégies argumentatives auxquelles a recours Sadate dans son discours pour effacer les traits négatifs de son ethos préalable en vue de se construire une image de soi favorable auprès de son auditoire qu'il veut être bien disposé à son égard. Khaled Mohy el-Dïn déclare que Sadate était «une personne qui a une compétence importante, c'est le fait de s'adresser aux publics, de comprendre leurs motifs et de leur parler de ce qu'ils veulent. [...] Dans tous ses rapports, il était très attentif à parler avec les gens ou même à les affronter en fonction de la prise de conscience de 


\section{Le conflit entre l'ethos préalable et l'ethos discursif dans le discours politique de Sadate prononcé à la Knesset israélienne}

leurs motivations. ${ }^{6} \gg$. Bien que Sadate soit considéré comme un orateur charismatique et fort et qu'il jouisse d'une compétence discursive distinguée, il est mis dans ce discours au défi de faire adhérer son auditoire aux arguments avancés.

Tout au long de son règne, Sadate prononce une centaine de discours dans et hors d'Egypte, mais ce discours est celui qui a soulevé le plus de controverses et de débats, à tel point qu'il est considéré un des grands discours du $\mathrm{XX}^{\text {ème }}$ siècle ${ }^{7}$, en plus, il se voit comme l'un des cent discours qui ont changé le monde ${ }^{8}$. Ce discours, se faisant dans un contexte particulier, a des incidences qui restent encore influentes jusqu'à présent dans la vie politique égyptienne et arabe.

\section{Contextualisation du discours en question}

En abordant la relation entre texte et contexte, les linguistes mettent toujours l'accent sur l'importance du dernier pour compléter et assurer la compréhension globale du premier ${ }^{9}$. En analysant la situation d'énonciation de ce discours, il nous apparait clairement que nous sommes devant un discours extraordinaire. Tout d'abord en ce qui concerne l'orateur, c'est le troisième président de la république égyptienne Anouar el-Sadate qui a posé les plans de la guerre d'octobre 1973, la guerre du Kippour, contre Israël. Le récepteur est les députés du peuple israélien. Quant au lieu et au temps, le discours est prononcé à la Knesset israélienne le 20 novembre 1977, peu d'années après cette guerre qui est faite essentiellement en réaction à une autre guerre ultérieure. On peut aussi brièvement recadrer historiquement ce discours d'après les points suivants :

- Du 6 au 25 octobre 1973 : la durée de la guerre d'Octobre.

- Lieu : Rives du Canal de Suez, péninsule du Sinaï et Plateau du Golan.

- Belligérants : coalition Égypte-Syrie contre Isrä̈l.

- 6 octobre 1973 : déclenchement surprise de la guerre le jour du jeûne de Yom Kippour, coïncidant avec la période du Ramadan, c'est ainsi la $4^{\text {ème }}$ guerre israéloarabe.

- 14 octobre 1973 : date charnière à partir de laquelle, passés le moment de surprise et les difficultés de l'armée israélienne, Tsahal reprend l'initiative. Retournement de la guerre à compter de cette date. Avant le cessez-le-feu, une division israélienne était arrivée à $101 \mathrm{kms}$ du Caire.

- 8 octobre 1973 : parallèlement à l'Égypte, à partir de cette date le vent tournera en faveur de Tsahal, après $15 \mathrm{~h}$ de combat.

- 22 octobre 1973 : Reprise par Israël de la position du mont Hermon.

- Ce qui vaut pour la guerre terrestre vaut pour la guerre navale : elle fut favorable à Israël qui s'imposa très nettement face à ses adversaires (en mer Rouge, face aux Égyptiens).

Il y eut de lourdes pertes (3000 tués pour 400000 hommes engagés du côté israélien/10 000 tués pour 1000000 d'hommes envoyés du côté arabe), ce qui a valu une crise du commandement israélien, crise de l'opinion et crise politique. 
Cessez-le-feu et accords intérimaires :

Pour les Arabes, et les Égyptiens en particulier, la conviction largement partagée fut alors que des négociations de paix pourraient permettre d'obtenir ce qui n'avait pu être gagné sur le terrain. Sadate, qui était entré en guerre pour récupérer le Sinaï, était contrarié par le rythme lent des négociations de paix. En novembre 1977, il fit un pas inattendu en effectuant un voyage officiel en Israël, devenant ainsi le $1^{\text {er }}$ leader arabe à reconnaître de facto l'existence d'Israël. Ceci donna lieu au Sommet de Camp David (5-17 septembre 1978) réunissant Sadate et Begin sous la houlette du président américain Jimmy Carter, aboutissant au traité de paix israélo-égyptien de 1979. Israël se retira du Sinaï en échange d'une normalisation et d'une paix durable avec l'Égypte ${ }^{10}$.

Donc, nous sommes devant un discours politique entre deux nations qui sont encore dans un état de guerre. Comment cette situation d'énonciation délicate permettra-t-elle d'entamer une entreprise de persuasion?

\section{Problématique et méthode de travail}

L'ethos préalable représente dans ce discours une problématique majeure étant donné que l'orateur est le président de l'État égyptien qui est en l'occurrence en cas de guerre avec l'État israélien représenté par l'auditoire à savoir les députés de la Knesset. Nous allons donc considérer l'argumentation comme « une pratique sociale $^{11} \gg$ tout en mettant l'accent sur le statut de l'orateur, sa relation sociale avec l'auditeur, les circonstances socio-historiques dans lesquelles il prend la parole et ainsi que la nature de l'auditoire visé.

Pour éclaircir les fonctionnements discursifs et explorer les stratégies argumentatives mises en œuvre dans le discours entre les mains, nous adoptons la méthode d'analyse argumentative qui se réclame à la fois de la rhétorique aristotélicienne et de l'analyse du discours. Nous nous servons en même temps d'une approche communicationnelle étant donné que, d'une part, l'effet de la situation de communication dans laquelle le discours est produit est indispensable pour analyser la visée ou la dimension persuasive d'un discours. D'autre part, les stratégies discursives employées par le sujet argumentant ne prennent pas sens si ce dernier ne respecte pas les conditions de la situation de communication ${ }^{12}$. Comment un président d'un état étranger, ou plutôt ennemi, peut-il convaincre un public qui n'est pas le sien et qui a préalablement de lui une image défavorable? En termes d'analyse du discours, comment peut-il s'adapter à son auditoire particulier? Quelles stratégies discursives doit-il adopter pour modifier son ethos préalable pour présenter une image de soi crédible et favorable? D'ailleurs, comment éviter de passer pour un traître aux yeux des peuples arabes? Voilà beaucoup de questions intéressantes auxquelles nous allons essayer de trouver des réponses. Commençons de prime abord par le lien délicat orateur / auditeur. 


\section{Le conflit entre l'ethos préalable et l'ethos discursif dans le discours politique de Sadate prononcé à la Knesset israélienne}

\section{La problématique de l'auditoire}

Soulignons d'abord que l'auditoire est, selon Perelman et Olbrechts-tyteca, «L'ensemble de ceux sur lesquels l'orateur veut influer par son $\operatorname{argumentation}^{13}$ ». D'après cette définition, nous pouvons dire que Sadate s'adresse dans son discours à un double public dont la première partie est présente et l'autre absente. Il vise d'une manière directe les députés de la Knesset, mais derrière ces derniers se profilent tous les Israéliens dont Sadate veut obtenir le soutien et l'adhésion à l'opinion défendue.

Ajoutons aussi que le public est une composante essentielle de l'argumentation parce que son approbation ou désapprobation est «la mesure du $\operatorname{discours}^{14} »$. L'adaptation à l'auditoire représente ici pour Sadate une grande difficulté parce qu'il ne s'adresse ni aux agents de son parti politique, ni à son propre peuple, ni même à un peuple ami. Comment entre-t-il en contact avec son auditoire et à travers quelle relation? On s'interroge ainsi sur « le processus de prise de contact. Il est clair que Sadate n'a pas d'autorité précise sur son auditoire afin qu'il puisse l'obliger à exécuter un ordre ou à l'orienter dans une certaine direction. Il essaye de persuader un auditoire qui n'a ni les mêmes valeurs, ni les mêmes objectifs que lui. Le discours se fait après 60 années de tensions entre Juifs et Arabes. La situation est ainsi compliquée et défavorable à l'orateur et à son entreprise de persuasion. Dans une telle situation critique, quelle stratégie argumentative pourrait être adoptée par l'orateur afin de mener à bien son processus de persuasion? Ruth Amossy propose une stratégie logique et efficace à ce propos : "L'interaction argumentative, si elle s'avère possible, doit alors se fonder sur des prémisses communes aux deux interactants au-delà de leurs divergences marquées ${ }^{15}$ »

C'est la même stratégie suivie par Sadate, mais il l'applique systématiquement à trois étapes. Premièrement, il met en exergue les prémisses communes entre l'auditoire et son propre peuple :

1-Vous voulez vivre avec nous dans cette région du monde. Moi, je vous dis tout sincèrement que nous vous accueillons parmi nous avec la pleine sécurité et la pleine sûreté ${ }^{16}$.

Soulignons qu'avec l'utilisation de l'adverbe sincèrement, l'orateur se met dans une obligation morale de réaliser ce qu'il dit. Deuxièmement, il met en relief des prémisses qui valorisent les intérêts d'Israël, la première en est la reconnaissance de l'Etat d'Israël :

2- J'ai annoncé à maintes reprises qu'Israël était devenu une réalité accomplie. 
En effet, avec ces «maintes reprises », le but de l'orateur n'est bien sûr pas de redonner ou de reconfirmer des informations dont l'auditoire est déjà au courant, mais il cherche à construire une représentation particulière de la réalité qu'il veut faire partager avec ce dernier en créant un effet d'insistance. L'énonciateur a ainsi une tendance vers l'intervention sur les opinions et les attitudes des allocutaires pour chercher l'adhésion de ces derniers. Ainsi Sadate adopte-il à son but argumentatif des éléments préalablement sélectionnés et qui peuvent servir de point de départ à l'argumentation. Puis, il touche une question primordiale pour le gouvernement et le peuple israéliens, c'est celle de la paix d'Israël.

3- Mais je vous dis aujourd'hui, devant le monde entier, que nous acceptons de vivre avec vous dans une paix juste et durable

Troisièmement, Sadate met en valeur des prémisses communes à l'humanité toute entière, y compris ipso facto les députés israéliens. Il valorise des valeurs comme celles de l'Homme, de la paix, de la justice ${ }^{17}$. L'orateur touche ainsi des causes sociales et politiques ayant une grande valeur auprès de son auditoire. Ici Sadate a recours à ce qu'on appelle « l'argumentation idéologique ${ }^{18}$ » qui est une partie de la communication politique. En cherchant les croyances et les valeurs de son public, l'orateur s'en fait ainsi une image qui se traduit verbalement dans son discours. Ce faisant, il construit parallèlement une image de sa propre personne s'adaptant avec celle du public. Les deux images sont donc reliées l'une à l'autre et se composent ensemble au fur et à mesure au sein du discours. Si l'orateur peut adapter, ou du moins rapprocher, son image à celle de son auditoire, il peut en même temps mettre en valeur l'image de soi et avoir ainsi plus d'adhésion auprès de ce dernier. Nous voulons à présent découvrir la démarche de Sadate vers la construction de l'image de lui-même.

\section{L'ethos ou l'image de soi}

En essayant de construire une image favorable de lui-même, l'orateur cherche explicitement ou implicitement à assurer sa légitimité, sa crédibilité et son autorité. Mais est-ce que le discours prononcé dans un moment donné suffit à lui seul pour faire connaître l'orateur et pour s'en faire une image pouvant orienter l'adhésion des esprits de son auditoire? En essayant de retirer la réponse de la situation de communication du discours en question, nous trouvons que l'orateur, ici, a déjà une image - défavorable - auprès de son public avant même la prise de parole et qui a bien entendu un impact sur la réception de son discours. Effectivement, en produisant une image de soi tout au long du discours, l'orateur ne doit pas oublier de prendre en compte les données préexistantes de sa personne connues du public, notamment son statut institutionnel. De là vient le rôle de l'image 


\section{Le conflit entre l'ethos préalable et l'ethos discursif dans le discours politique de Sadate prononcé à la Knesset israélienne}

dérivée d'une connaissance préalable de la personnalité de l'orateur, c'est-à-dire l'ethos prédiscursif. Nous étudions ainsi l'image de soi de l'orateur à deux niveaux: l'image préexistante, l'ethos préalable ou prédiscursif, et l'image qu'il construit dans son discours, l'ethos discursif.

\subsection{Ethos prédiscursif ou préalable}

En se fondant sur des éléments préexistants, l'ethos préalable coïncide dans le discours politique avec la fonction institutionnelle de l'orateur, dite l'ethos institutionnel qui est basé sur le « rôle que remplit l'orateur dans l'espace social (ses fonctions institutionnelles, son statut et son pouvoir), mais aussi sur la base de la représentation collective ou du stéréotype qui circule sur sa personne ${ }^{19}{ }{ }$.

En effet, l'ethos institutionnel joue avec la représentation collective de Sadate à former son ethos préalable. La difficulté consiste bien sûr au fait que cet ethos prédiscursif va à l'encontre du but argumentatif de l'orateur. Nous allons étudier les stratégies argumentatives auxquelles l'orateur a recours pour produire une impression conforme à son processus argumentatif.

\subsection{Le retravail de l'ethos préalable}

Bien que le statut institutionnel de Sadate aille contre sa visée argumentative, il le prend comme un point de départ pour, dans un premier temps, se donner de la crédibilité; dans un deuxième temps, modifier son image prédiscursive. Il nous semble que la stratégie principale de Sadate s'articule sur un axe principal qui consiste à retravailler son ethos préalable pour chercher à obtenir plus de crédibilité. Pour ce faire, Sadate adopte plusieurs stratégies discursives dont la première se base sur la justification de la raison d'être de son discours lui-même.

\subsubsection{Les motivations du discours}

La décision de la visite de Sadate en Israël, et par la suite son discours, était une surprise autant pour les Israéliens que pour les Arabes, tout comme pour les Égyptiens eux-mêmes. Soulignons aussi que par cette visite, Sadate va contre l'idéologie consensuelle arabe en l'occurrence. Malgré le fait qu'il se dresse en porteparole de tous les Arabes, il ne se soumet ainsi pas au stéréotype de la société arabe qui refusait toute sorte de normalisation des relations avec Israël. Par ailleurs, avec la déclaration de Sadate de son intention de rendre visite en Israël pour chercher la paix, les Israéliens pensent que c'est une tactique politique à travers laquelle il veut les mettre en situation critiquable devant l'opinion publique mondiale. Son cas est donc problématique : aux yeux des Arabes, il est considéré pour ainsi dire comme un traitre politique; en ce qui concerne les Israéliens, il est perçu comme un 
tacticien politique. Il incombe donc à Sadate de défendre sa thèse, la cause de ces doubles accusations, pour consolider sa crédibilité. Pour légitimer sa prise de décision, il a recours à un certain «discours de justification ${ }^{20} »$. La première partie du discours est ainsi focalisée sur le pourquoi de la visite; l'orateur justifie sa prise d'initiative de visiter Israël en montrant à la fois que cette décision ne lui était pas facile et qu'il l'a prise après une longue réflexion. Dans cette partie, l'ethos de Sadate se construit implicitement à travers l'affichage de ses motivations. Au préalable, il annonce clairement la raison d'être de la visite :

4- Aujourd'hui, je suis venu vers vous sur deux pieds assurés, afin de construire une nouvelle vie et d'établir la paix.

Son but est bien entendu louable pour son auditoire avec qui il veut « construire une nouvelle vie ». Il commence ainsi la première étape pour effacer l'image préalable du guerrier. Il veut complètement tourner la page des tensions entre ces deux nations étant, depuis soixante années, en cas de guerre. A ce moment-là, la construction de la paix sera possible. Puis, Sadate montre que sa décision est toute personnelle

5- Au sujet de cette décision, je n'ai pas consulté mes collègues et frères, chefs des Etats arabes ou des Etats de confrontation. Il y en a qui m'ont appelé et m'ont exprimé leur opposition après l'annonce de ma décision.

Sadate annonce aussi l'élaboration efficace de cette décision qu'il a prise après une longue réflexion et qu'il considère comme un grand risque, mais qui lui est imposée vu la responsabilité qu'il assume. L'orateur déclare qu'il se rend en Israël non seulement pour chercher les intérêts arabes, mais aussi pour chercher ceux d'Israël. Il veut protéger le citoyen arabe ainsi que le citoyen israélien :

6- Je suis venu chez vous pour construire ensemble la paix permanente et juste, afin de ne pas laisser verser une seule goutte de sang d'un corps arabe ou israélien.

Sadate exprime sa volonté d'agir au service de la paix pour tous. La décision est donc personnelle, bien étudiée et prenant en considération le bien public d'Israël aussi que des Arabes. Il se déclare ainsi poussé par la responsabilité dont il est chargé; il partage les mêmes sentiments de peur envers l'auditoire. Par cette inauguration de discours, il nous semble qu'il veut se présenter comme un homme fidèle, sage et digne de confiance. Il est clair que Sadate cherche de la crédibilité auprès de son auditoire. 


\section{Le conflit entre l'ethos préalable et l'ethos discursif dans le discours politique de Sadate prononcé à la Knesset israélienne}

\subsubsection{L'enjeu de la crédibilité}

La crédibilité est une notion clé dans l'analyse de l'ethos parce que, comme le dit Amossy, l'ethos est «l'image que l'orateur construit de sa propre personne pour assurer sa crédibilité ${ }^{21}{ }^{22} \gg$. Dans cette perspective, après avoir justifié la décision, l'orateur cherche à se faire une image crédible. Pour présenter les preuves de sa crédibilité, le sujet argumentant doit construire son «identité discursive » dans deux directions : le « dire vrai » et le « dire juste» :

«Le «dire vrai» suppose que le sujet qui parle dise ce qu'il pense sans maquillage aucun. Si l'on sait que ce qu'il dit correspond à ce qu'il pense, on dira qu'il est sincère et digne de foi. Le «dire juste» suppose que l'on puisse créditer le sujet qui parle de sérieux et d'honnêteté dans ses affirmations, déclarations, explications ${ }^{23}{ } »$.

En effet, ces deux directions argumentatives sont bien présentes tout au long du discours en question. Sadate essaie de s'attribuer, soit explicitement ou implicitement, quelques qualités dont le but discursif est de renforcer sa crédibilité auprès de l'auditoire. La première en est le fait qu'il est capable d'assumer la responsabilité, terme qui revient plusieurs fois dans le discours:

7- Et ceux qui assument, comme nous, cette responsabilité imposée, sont les premiers à avoir le courage de prendre des décisions fatidiques correspondant à la grandeur de la situation.

Sadate se déclare capable de prendre «des décisions fatidiques », même dans les moments difficiles. Il est aussi toujours attaché aux exigences de la responsabilité; il se déclare engagé à prendre la responsabilité de réaliser la paix :

8- J'ai assumé, et assume encore, les exigences de la responsabilité historique.

Le fait de jouer sur l'engagement s'insère clairement dans l'enjeu de la crédibilité. Il annonce prendre ses décisions et effectuer ses actes en vue de la responsabilité qui lui est attribuée. Il s'attache ainsi à la paix pour réaliser sa responsabilité envers tous les pays arabes. Il va plus loin en assurant sa responsabilité envers les Israéliens, voire envers toute personne sur terre :

9- Si je dis que je veux éviter à tout le peuple arabe les maux de nouvelles guerres catastrophiques, je déclare, devant vous, en toute sincérité, que j'éprouve les mêmes sentiments et tiens la même responsabilité pour tout homme dans le monde, et certainement envers le peuple israélien. 
Cet extrait du discours laisse voir un aspect de sa personnalité positif aux yeux de l'auditoire : il a parallèlement peur pour le peuple arabe et le peuple israélien. Il se charge de toute la responsabilité possible pour se montrer doué de bon sens. Par cette présentation de soi, Sadate entend montrer qu'il peut se poser dans une relation d'amitié avec son auditoire et orienter ainsi le discours vers la construction d'un ethos répondant aux attentes de ce dernier. En plus, il élargit l'extension de sa responsabilité pour comprendre les générations futures :

10- Pour tout cela, et pour protéger la vie de tous nos enfants et frères, $[\ldots]$ pour notre responsabilité envers les générations à venir, pour le sourire de chaque enfant né sur notre terre, pour tout cela, j'ai pris ma décision de venir chez vous, malgré tous les risques, afin de prononcer mon discours.

Il envoie ici sa responsabilité au maximum en faisant, en même temps, abstraction de tous les avertissements qui auraient pu l'empêcher de prononcer son discours. Il sous-entend ainsi qu'il respecte les exigences de la responsabilité imposée en dépit de tous les défis parce que son but ultime est la réalisation de la paix pour protéger les intérêts de tous. Il est donc complètement attaché aux valeurs de la paix et opposé à la violence. Il se déclare doué du sens des responsabilités et du devoir envers les peuples égyptien, arabe et israélien, mais aussi envers l'humanité toute entière. Et en cherchant une position plus crédible, il mêle la responsabilité à l'audace :

11- Personne n'imaginait que le président du plus grand pays arabe, le responsable premier et le plus grand de la cause de la guerre et de la paix dans la région du Moyen-Orient, pourrait se déclarer prêt à aller sur la terre de l'adversaire, alors que nous étions en état de guerre.

Le fait que l'Egypte soit le «plus grand pays arabe » est une technique discursive visant à attribuer la même qualité à la personnalité de l'orateur elle-même puisqu'il se présente en tant que responsable de ce grand pays. Par cette présentation de soi, il vise à persuader son auditoire de sa crédibilité et de sa légitimité. C'est seulement avec et par lui qu'on peut engager une paix réelle avec tous les Arabes. Notons qu'il utilise des adjectifs évaluatifs et axiologiques, /al $\square$ akbar/ le plus grand, /al $\square$ olā/ première, pour renforcer le contenu de ses énoncés.

D'autre part, il se présente sous un jour clair et authentique. Le taux de fréquence du mot /ṣedq/, authenticité, est élévé. Par cette qualité, il qualifie son acte même d'énonciation :

12- Je vous déclare en toute authenticité [...] Je déclare devant vous en toute authenticité [...] J'aimerais aussi vous avertir en toute authenticité... 


\section{Le conflit entre l'ethos préalable et l'ethos discursif dans le discours politique de Sadate prononcé à la Knesset israélienne}

Il adopte aussi la posture d'un homme tolérant en annonçant le pardon pour ceux qui ont surpris ou se sont doutés de son intention de visiter Israël.

13- Je cherche une excuse pour tous ceux qui ont accueilli ma décision avec surprise voire avec stupéfaction quand je l'avais annoncée au monde entier devant le Conseil du Peuple égyptien [...]

Il a apporté ainsi une autre preuve de sa crédibilité en reconnaissant l'existence possible d'autres points de vue. Il prononce un énoncé à valeur illocutoire d'excuse et de justification en utilisant le verbe /? ?altamis/, " chercher une excuse » qui, en plus de la présentation du pardon par nature, montre à la fois son désir sincère de trouver des justifications en face de l'accusation qui lui est adressée par quelquesuns. Par ce faire, il offre ainsi l'image d'une personne qui veut convaincre de sa volonté de la paix et de l'intérêt général de tous en faisant abstraction de toute vengeance personnelle éventuelle.

Ainsi Sadate essaie-t-il fortement de retravailler l'image préalable que l'auditoire se fait de lui. C'est en essayant de gommer les traits négatifs de cette image que l'orateur part vers la construction d'un ethos favorable. En confirmant sa responsabilité vers son auditoire, sa clarté et sa tolérance, il peut en grande partie avoir plus de crédibilité et être digne de la confiance de son auditoire. Soulignons à ce propos que la responsabilité et la clarté sont des exigences fréquentes non seulement chez Sadate, mais aussi, comme le constate Robrieux chez tous les orateurs contemporains $^{24}$. Il nous semble que les politiciens estiment, d'après leur expérience, que ces deux qualités touchent bien l'auditoire et servent à augmenter leur crédibilité. Les énoncés exemples cités mettent en relief certaines qualités de la personnalité de l'orateur qui inspirent la confiance à l'auditoire et lui confèrent de la crédibilité. Il se présente comme un homme d'action, crédible et attaché à la cause de la paix.

Or, l'ethos d'un politicien, nous semble-t-il, ne se limite pas à la présentation directe de soi, mais il s'avère aussi clair dans le choix des mots et l'efficacité des arguments choisis ${ }^{25}$. Dans les lignes qui suivent, nous voulons découvrir les arguments dont Sadate se sert pour achever son processus argumentatif.

\subsection{Argument d'autorité}

L'argument d'autorité consiste à faire admettre la thèse qu'on défend en s'appuyant sur une autorité digne de foi. Celle-ci doit, par conséquent, être admise par l'auditoire. Dans le discours politique, l'autorité est primordiale parce qu'elle renforce son influence et y apporte de la crédibilité. En vue de consolider sa légitimité et sa crédibilité, cette catégorie d'arguments est bien fréquente dans le discours de Sadate. Cette stratégie argumentative a deux aspects : soit l'orateur a recours à sa propre autorité, soit il convoque une autre autorité extérieure. Le deuxième aspect est bien sûr plus utile pour notre orateur car sa crédibilité est jusque-là cachée ou remise en cause aux yeux de son public. 


\subsubsection{L'autorité extérieure}

En cherchant de la crédibilité et pour renforcer l'influence que son discours peut avoir sur le public, l'orateur se réfère à des personnalités publiques célèbres. Il nous paraît que Sadate est bien prudent en choisissant des citations qui répondent bien à la culture et aux croyances sociales de son auditoire. La première citation prononcée appartient à Ghandi, considéré comme une personnalité digne de foi et dont la citation a pour objectif de renforcer son argument parce que les grands hommes ne pourraient pas se tromper. Ghandi - homme politique, philosophe et révolutionnaire indien - était pour la non-violence, la fraternité et la paix entre les différentes communautés. La citation d'une telle personne sert bien à confirmer la visée argumentative dans le contexte de ce discours où l'orateur cherche à établir une paix juste et durable entre deux nations. Au début du discours, en abordant l'influence négative de la guerre sur l'Homme, Sadate clôt ces énoncés par cette citation de Ghandi :

14- L'Homme que Dieu a créé, comme l'a dit Gandhi, l'apôtre de la paix, « pour marcher sur ses deux pieds, construire la vie et adorer Dieu. »

Si en produisant une citation d'autorité, on suppose que l'orateur " s'efface donc devant un «locuteur» superlatif qui garantit la validité de l'énonciation ${ }^{26}$ », il nous semble que Sadate ne veut pas s'effacer, mais, au contraire, il veut aller plus loin en s'inscrivant complètement dans l'idéologie ghandienne, et ce en reprenant les mêmes mots que Ghandi en décrivant son voyage en Israël :

15- Aujourd'hui, je suis venu vers vous sur deux pieds assurés, afin de construire une nouvelle vie et d'établir la paix. Nous tous, sur cette terre, la terre de Dieu, nous tous, musulmans, chrétiens et juifs, adorons seulement Dieu. Les enseignements et les commandements de Dieu sont un amour, une authenticité, une pureté et une paix.

D'autre part, Sadate essaie d'ancrer son discours dans la culture des récepteurs. La preuve en est la citation des textes puisés dans la Thora et l'allusion aux prophètes, fils d'Israël. Bien que le Coran et la Sunna, c'est-à-dire la culture de l'énonciateur, soient pleins de textes qui servent bien à renforcer son point de vue ${ }^{27}$, Sadate s'en débarrasse pour, d'un côté, annoncer son respect de la culture réceptrice et, d'un autre côté, sous-entendre qu'il partage les mêmes valeurs qui prônent la paix et la fraternité.

Il est frappant de souligner que les discours politiques de Sadate jouissaient d'un ancrage religieux indéniable ${ }^{28}$, ancrage qui se faisait bien sûr dans la religion musulmane étant donné que ces discours sont adressés aux égyptiens. Or ce n'est pas le cas pour le discours, objet de notre étude. Là où l'ancrage se fait dans la culture de l'auditoire, la culture juive. Il fait référence plusieurs fois à la Bible. Citons, entre autres, cet extrait où il cite le prophète Salomon dans une question rhétorique : 


\section{Le conflit entre l'ethos préalable et l'ethos discursif dans le discours politique de Sadate prononcé à la Knesset israélienne}

16- Pourquoi ne croyons-nous pas en la sagesse du Créateur citée dans les sentences de Salomon: La trahison est dans le cœur de ceux qui pensent au mal. Quant aux missionnaires de la paix, ils ont une joie. »

Cet extrait de la Bible, contient une comparaison entre ceux qui prônent la paix et ceux qui pensent au mal. Aussi, vers la fin du discours, il annonce qu'il répète le propos de Zacharie:

17- Mon Dieu, je répète avec Zacharie son propos : «Aimez le droit et la paix ».

En empruntant ces énoncés, qui appuient ses arguments, à la Bible, l'orateur a recours à ce que les analystes de discours appellent "la citation-culture [qui provoque] une adhésion presque automatique ${ }^{29}$ " et ce pour accéder plus rapidement à l'adhésion des esprits des allocutaires.

Soulignons, à ce propos, que Sadate utilise aussi des termes empruntés à la culture biblique: Cantique, Hymne. Quant à la fin du discours, il retourne à sa propre culture en citant un verset coranique dont le contenu appelle au rapprochement entre les nations ; c'est le verset 84 de la sourate La famille d'Imrān :

18- Je cite des versets coraniques celui où Dieu dit : « Dis : Nous croyons en Allah, à ce qu'il nous a révélé, à ce qu'on a révélé , à ce qu'Il a révélé à Abraham, à Ismaël, à Isaac, à Jacob et aux Tribus, et à ce qui a été donné à Moïse, à Jésus et aux prophètes de la part de leur Seigneur. . Nous ne faisons aucune distinction entre eux, et c'est à Dieu que nous sommes soumis. »

L'orateur fait ainsi appel à une autorité incontestable pour le revêtir de la crédibilité, en essayant, en même temps, que ce revêtissement soit puisé dans la culture de l'auditoire pour être plus influent. En prenant appui sur la religion, l'orateur se construit un ethos d'un homme crédible et authentique, parce que celui qui se réfère à Dieu entraîne une certaine adhésion. De plus, cet ethos de crédibilité aide à casser l'ethos préalable parce qu'un homme crédible et croyant ne pourrait pas s'attaquer à ceux avec qui il s'engage dans un processus de paix juste et durable.

Nous voulons à présent découvrir l'autre aspect de l'argument d'autorité; à savoir l'autorité de l'orateur.

\subsubsection{Le recours à sa propre autorité}

Avant d'envisager cet aspect de l'argument d'autorité, soulignons que celuici n'a pas la même influence que la citation référée à une personne autre que l'orateur. Quand même, cet aspect personnel de l'argument d'autorité est utile jusqu'à un certain point pour Sadate par ce qu'il veut s'adresser à son auditoire sur la base de son identité sociale comme un président d'un pays pour attribuer à sa parole plus de légitimité. Ainsi met-il en exergue à maintes reprises sa position sociale en tant que chef d'Etat: 
19- Personne n'imaginait que le président du plus grand pays arabe, le responsable premier et principal de la cause de la guerre et de la paix dans la région du MoyenOrient, pourrait se déclarer prêt à aller sur la terre de l'adversaire, alors que nous étions en guerre.

Il cherche donc à valoriser son identité sociale pour confirmer le relais de son discours. D'ailleurs, nous pouvons insérer dans cet aspect de l'argument d'autorité, l'argument $d u$ témoignage $e^{30}$ qui consiste au fait d'avoir été témoin d'une manifestation ou d'un événement. En ce qui concerne les relations araboisraéliennes, l'orateur se met à raconter une expérience personnelle:

20- Nous vous refusions certainement et nous avions nos causes et motifs... Oui.

Nous refusions bien sûr de vous rencontrer partout... Oui.

Nous vous décrivions certes comme Israël prétendu... Oui.

Cet argument vise à conférer plus de crédibilité à son propos et à souligner qu'il est de parti pris dans ces événements. Comme témoin, il décrit les maux subis à cause du mur de la ligne Bar-Lev :

21- Il y avait certes entre vous et nous un grand mur, que vous aviez essayé de construire tout au long d'un quart de siècle, mais il a été détruit en 1973.

C'était un mur au profit d'une guerre psychologique de plus en plus croissante et grandissante.

C'est l'orateur lui-même qui a conduit la guerre de 1973, là où ce mur est détruit. De même, poussé par la force de cet argument du témoignage, l'orateur ose suggérer à son auditoire d'écraser aussi le mur psychologique, fait à la suite de ce mur détruit :

22- Nous devons avouer ensemble que ce mur est tombé et détruit en 1973. Pourtant, il reste un autre mur constituant une barrière psychologique complexe entre vous et nous. [...] Je vous demande aujourd'hui, à cause de ma visite chez vous, pourquoi ne pas étendre nos mains avec foi et sincérité pour détruire ensemble cette barrière?

Il se présente ainsi en tant qu'investisseur d'un certain statut (autorité) qui lui permet d'indiquer à l'avance ce qui devrait être fait ou dit. En plus, il est frappant de souligner que l'orateur relie sa propre autorité provenant de son statut social à l'argument du témoignage, et ce dans un passage compatissant adressé à tout le peuple israélien:

23- Annoncez à vos enfants que la guerre passée est la dernière et la fin des maux, et que ce qui est à venir est le nouveau début de la nouvelle vie, celle d'amour, de bien, de liberté et de paix.

Ô mère, qui êtes endeuillée, 


\section{Le conflit entre l'ethos préalable et l'ethos discursif dans le discours politique de Sadate prononcé à la Knesset israélienne}

ô femme, qui avez perdu votre mari,

ô fils, qui avez perdu un frère et un père,

ô toutes les victimes des guerres,

remplissez la terre et l'espace des hymnes de paix...

Son statut de la présidence lui permet de prévoir que la guerre de 1973 sera la dernière entre Israël et les pays arabes. En mobilisant ainsi la source de sa légitimité, il fait appel à des catégories sociales différentes du peuple israélien dont les maux sont attestés par lui-même et ceci dans le but de leur demander de s'attacher à la paix et à l'espoir.

Nous constatons que la mise en valeur de sa position sociale ainsi que la citation des événements que l'orateur raconte en tant que témoin viennent consolider la crédibilité de ce dernier. Après avoir mis l'accent sur l'autorité dans l'argumentation, il est intéressant de finir par montrer qu'avec celle-ci, il s'agit toujours du champ de l'argumentation et non pas de celui du pouvoir. "L'autorité en argumentation n'est finalement qu'une proposition faite à l'autre, libre d'en disposer $^{31}$ ». Sadate lui-même semble reconnaître cette vérité dans l'argumentation, et ce en annonçant vers la fin de son discours qu'il a fait son devoir en venant en Israël et c'est à son auditoire d'agir comme lui en vue de la construction de la paix :

24- [...], même de venir à Jérusalem, pour m'adresser aux députés de la Knesset, les délégués du peuple israélien, avec tous les faits qui couvent dans mon esprit. Puis, je vous laisserai décider pour vous-mêmes, et que Dieu fasse ce qu'll veut.

\subsection{L'argument de communauté}

En cherchant à faire adhérer les esprits de l'auditoire à sa visée argumentative, Sadate essaie de trouver une communion en faisant appel à certaines valeurs communes ou à des vérités universelles. Il s'agit ici d'appuyer ses arguments sur des éléments déjà reconnus et acceptés par les allocutaires.

« Être d'accord à propos d'une valeur, c'est admettre qu'un objet, un être ou un idéal, doit exercer sur l'action et les dispositions à l'action une influence déterminée,[...] L'existence des valeurs, comme objets d'accord permettant une communion sur des façons particulières d'agir, est liée à l'idée de multiplicité des groupes ${ }^{32} 》$.

A l'instar de Pereleman, Robrieux divise les valeurs en valeurs concrètes et valeurs abstraites, en annonçant que ces dernières "peuvent être universelles, c'està-dire admises par tout homme quels que soient l'époque et le lieu considérés ${ }^{33}{ }^{3}$. De même, Sadate se sert de cet ensemble de valeurs dans la recherche d'un consensus. D'autre part, le recours aux valeurs abstraites cadre avec d'autant plus de force avec son projet argumentatif, étant donné qu'avec ce discours entre les mains, l'orateur 
veut opérer des modifications radicales dans la démarche des relations araboisraéliennes. Sadate a ainsi un «esprit révolutionnaire » qu'il veut montrer dans ce discours. La plus importante valeur commune sur laquelle Sadate s'appuie essentiellement pour développer son argumentation et qui semble justifier, d'une manière ou d'une autre, le motif de sa visite en Israël, c'est celle de la vie. La protection de celle-ci contre les maux de la guerre n'est pas restreinte à une communauté donnée. Consultons cet extrait où Sadate met en valeur la vie de l'être humain.

25- La victime de la guerre est l'homme. L'esprit perdu dans la guerre est celui d'un homme, qu'il soit arabe ou israélien. La femme qui devient veuve est un être humain dont le droit est de vivre dans une famille heureuse, qu'elle soit arabe ou israélienne

Ainsi le locuteur choisit-il l'argument qui pourrait faire écho auprès de son auditoire. Il part des présupposés communs sur lesquels tout le monde est d'accord. L'orateur veut ainsi mettre en valeur «la vie», quel qu'en soit le détenteur, en faisant abstraction de la nationalité et de la religion, et dont la perte a un impact négatif sur l'humanité. L'orateur insiste de plus en plus sur cette valeur en déclarant qu'elle est la raison d'être de son discours :

26- Pour tout cela, et pour protéger la vie de tous nos enfants et frères, [...] en vue de l'évolution de l'homme, de lui faire plaisir et de lui donner le droit à une vie décente, [...]pour tout cela, j'ai pris ma décision de venir chez vous, malgré tous les risques, afin de prononcer mon discours.

Il surajoute aussi qu'il cherche à établir la paix juste pour sauver toute vie, soit en Israël, soit dans les pays arabes. En utilisant une question rhétorique provocatrice, dont l'objet est de déclencher une réaction chez un adversaire piqué au vif $^{34}$, il s'exclame des résultats malheureux de tuer les âmes et ce pour faire peur à son auditoire.

27- Pourquoi laisserions-nous comme héritage aux générations futures les résultats de bains de sang, de la perte de vies, des enfants orphelins, des veuves, de la destruction des familles et des gémissements des victimes?

D'ailleurs, il nous semble que ce n'est pas par hasard que Sadate se concentre sur des mots à valeur pathétique comme « victimes », « sang », « guerre ». En utilisant de tels mots, il cherche à créer une certaine stratégie psychologique d'influence sur son auditoire pour augmenter la force d'adhésion. Nous constatons que l'orateur s'attarde beaucoup sur la question de la vie et les conséquences malheureuses qui résultent de sa perte. La protection de la vie est bien entendu une cause défendue et soutenue par tous les peuples et les Organisations nationales et internationales. Il confère ainsi à ses arguments une certaine dimension d'universalité.

Nous pouvons aussi rencontrer un autre élément relevant de l'argument de communauté, à savoir les maximes qui constituent avec les proverbes des manières 


\section{Le conflit entre l'ethos préalable et l'ethos discursif dans le discours politique de Sadate prononcé à la Knesset israélienne}

commodes d'exprimer des valeurs ou des vérités immuables ${ }^{35}$. Sadate choisit quelques maximes qu'il considère comme pouvant bien servir son projet argumentatif. Nous en citons donc les suivantes :

28- Dans la vie des peuples et des nations, il y a des moments où les hommes ayant une vision sage doivent regarder au-delà du passé, de ses complexités et de ses sédiments, pour un nouveau départ vers de nouvelles perspectives.

En se servant de cette maxime, à savoir la vision sage de quelques hommes, considérée comme un argument typique valide en toute circonstance, Sadate pousse ses allocutaires à prendre l'initiative vers la construction de la paix afin qu'ils soient parmi ceux qui ont « une vision sage ». Il y a aussi une maxime qui se présente deux fois dans le discours :

29- il n'y a pas de bonheur pour quiconque au prix du malheur d'autrui.

Avec cette opinion commune basée sur le principe d'égalité, l'orateur essaie de convaincre ses allocutaires de permettre aux palestiniens d'établir leur état. Il veut ainsi les placer sous l'autorité de normes générales pour qu'ils avouent le droit du peuple palestinien. En suivant la même stratégie argumentative, l'orateur essaie de convaincre son auditoire d'exploiter l'opportunité de sa visite et d'affronter courageusement le problème palestinien:

30- on ne peut jamais résoudre un problème par l'évasion ou l'orgueil.

En prenant ainsi l'accord préalable comme point de départ de l'argumentation, l'orateur fait appel à des valeurs suprêmes et non pas à des intérêts personnels pour arriver plus facilement à l'adhésion de son auditoire. L'accord préalable joue ainsi un rôle indispensable dans la technique argumentative. "[A]rgumenter ne consiste pas seulement à mettre en forme, à couler son opinion dans le moule d'un argument, mais surtout, à appuyer cet argument sur un élément déjà accepté par l'auditoire ${ }^{36}$ "

Ainsi, c'est toujours dans un climat de valeurs et de croyances collectives que l'orateur tente de consolider ses points de vue pour réussir son projet argumentatif. En effet, cette relation d'empathie qu'il veut engager avec son public donne plus de valeur argumentative à son ethos et incite ce public à entrer dans l'action. L'ethos est ainsi un enjeu important dans les stratégies discursives parce que dans tous les discours politiques, l'enjeu suprême est, comme le dit Mayaffre, " d'affirmer l'identité d'un orateur pour favoriser l'identification d'un auditoire ${ }^{37}$ ».

\section{Conclusion}

A travers l'analyse de ce discours, nous pouvons observer à quel point l'orateur semble chercher, dans sa démarche argumentative, à présenter une image de soi digne de confiance pour assurer sa crédibilité et son autorité. Pour ce faire, l'orateur est confronté ici à une autre image préalable basée sur des éléments préexistants et ayant une relation étroite avec sa fonction institutionnelle. Cette 
dernière image va à l'encontre de la première; c'est pourquoi, Sadate fait de son mieux pour retravailler son ethos préalable. Dans son projet de modifier son ethos prédiscursif auprès de son public, l'orateur part de la clarification de la situation politique en l'occurrence entre son pays et Israël. Il semble ainsi maîtriser l'habilité de la communication politique dont "l'enjeu est la maîtrise de l'interprétation politique de la situation ${ }^{38}{ }$. Il essaie de s'adapter à son public pour pouvoir acquérir le soutien de sa cause. Sa stratégie argumentative réside principalement dans le remaniement de son ethos préalable pour gommer les traits négatifs de son image préalable et se donner en même temps une image favorable.

En justifiant et défendant sa décision de visiter Israël, il se montre comme une personne motivée qui peut tenir sa parole et réaliser des actions fortes en gardant l'intérêt de son propre peuple, sans néanmoins faire abstraction de celui de son auditoire. Et pour donner plus de crédibilité à son image personnelle, il a recours à une autorité extérieure en citant des personnalités célèbres comme Ghandi. Aussi fait-il référence à des prophètes comme Zacharie et Soliman. Il cherche aussi à évoquer certaines valeurs communes pour faire adhérer les esprits à sa visée argumentative.

Par ces quelques lignes, nous avons essayé de mettre en relief les stratégies argumentatives dans un discours politique assez différent des discours politiques habituels des chefs d'Etat ou même des autres discours de Sadate lui-même. Là où l'orateur s'oriente vers un auditoire adversaire dans des circonstances sociohistoriques nuisant à tout projet argumentatif. En dernier lieu, nous voulons annoncer que la porte est bien entendu ouverte pour chercher et analyser les stratégies argumentatives de tels discours où la relation orateur-auditoire est délicate.

\section{Le conflit entre l'ethos préalable et l'ethos discursif dans le discours politique de Sadate prononcé à la Knesset israélienne}

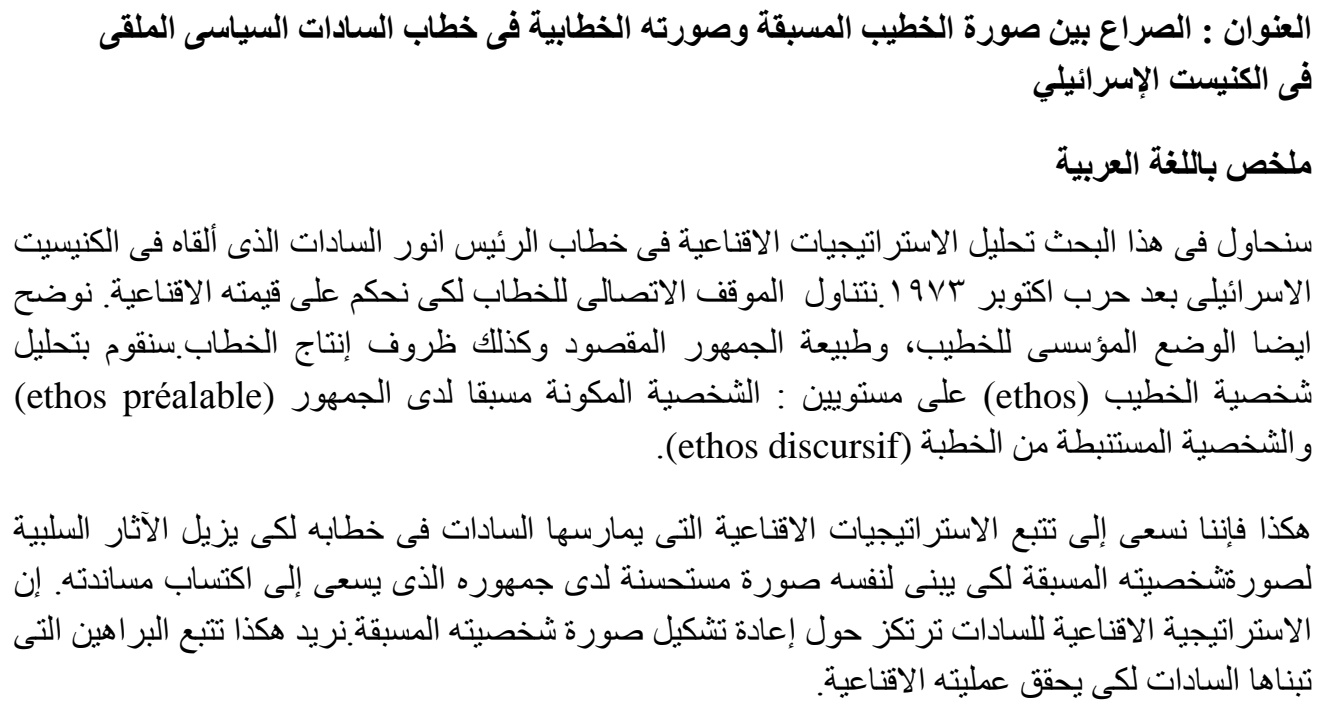




\section{Le conflit entre l'ethos préalable et l'ethos discursif dans le discours politique de Sadate prononcé à la Knesset israélienne}

\section{Résumé en français}

Dans cet article, nous essayons d'analyser les stratégies argumentatives dans un discours politique prononcé par le président égyptien Anouar Al-Sadate dans la Knesset israélienne après la guerre d'Octobre 1973. Nous mettons en relief la situation de communication du discours en question pour bien juger sa valeur argumentative. Nous illustrons le statut institutionnel de l'orateur, la nature de l'auditoire visée et les circonstances de la production du discours. L'ethos est analysé à deux niveaux : l'ethos préalable et l'ethos discursif.

Nous cherchons ainsi à retracer les stratégies argumentatives que mène Sadate dans son discours pour effacer les traits négatifs de son ethos préalable en vue de se construire une image de soi favorable auprès de son auditoire qu'il veut bien disposer à son égard. La stratégie argumentative de Sadate s'articule ainsi autour du retravail de son ethos préalable; nous voulons ainsi retracer les arguments que Sadate adopte pour achever son processus argumentatif.

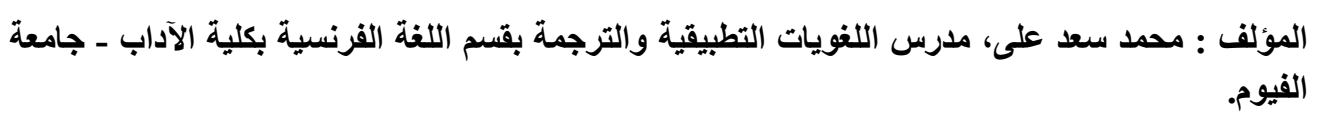

L'auteur : MOHAMED SAAD ALI, maître de conférences en linguistique appliquée et traductologie au département de Français à la faculté des Lettres - Université du Fayoum, Egypte 


\section{Notes}

1 . Je tiens à remercier Mme Marie-Anne Paveau, professeure de linguistique à l'université Paris 13, pour sa lecture attentive et ses remarques pertinentes.

2 . HERMAN, Thierry, « L'analyse de l'ethos oratoire », Philippe LANE (dir.), Des discours aux textes : modèles et analyses, Publications des universités de Rouen et du Havre, 2005, p.157-182. Et, AMOSSY, Ruth, « L'ethos et ses doubles contemporains. Perspectives disciplinaires ", Langage et société, V.3/149, 2014, p. 13-30.

3 . Les premiers travaux sont : MAINGUENEAU D., « Ethos, scénographie, incorporation », in Ruth AMOSSY. (dir.), Images de soi dans le discours, Lausanne, Delachaux \& Niestlé, 1999, P. 75-100.

Et

MAINGUENEAU D., « Problèmes d'ethos », Pratiques 113-114, Metz, Cresef, 2002, P. 5567.

4. AMOSSY, Ruth, KOREN, Roselyne, « Argumentation et discours politique » Mots. Les langages du politique, Trente ans d'étude des langages du politique (1980-2010), Nº 94 novembre 2010, 2010, p. 13.

5. CHARAUDEAU, Patrick, MAINGUENEAU, Dominique, Dictionnaire d'analyse $d u$ discours, Paris, Seuil, 2002, p. 239.

6. Khaled Mohy El-Din, /Wal $\square \bar{a} n \square$ atakalam/ [Et maintenant je parle], le Caire, le Centre d'Al-Ahram de traduction et de publication, 1992, p.238. Le texte original :

(شخص بيتلك مقدرة هامة ؛ وهي التوجه للجماهبر، وفهم نوازعها ومخاطبتها بما تريد [...] وكان في كل تعاملاته

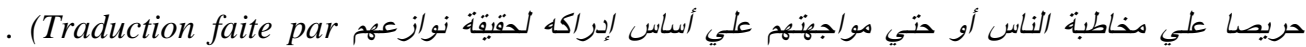
l'auteur de cet article )

7. BOUTIN, Christophe, 2009, Les grands discours du XXe siècle, Paris, Flammarion, 2009.

${ }^{8}$. MONTEFIORE, S. S., Ces grands discours qui ont changé le monde, de Jésus à Obama, traduit de l'anglais par Dominique Piolet-Françoise, Paris, Dunod, 2010.

9. ADAM, Jean-Michel, 2008, La linguistique textuelle, Armand Colin, Paris, P. 39.

${ }^{10}$. Pour recadrer le discours de Sadate, on peut se référer à plusieurs sources en arabe. En français, on peut consulter, par exemple, Encyclopédie Larousse.

${ }^{11}$. CHARAUDEAU, Patrick, « De l'argumentation entre les visées d'influence de la situation de communication », Christian BOIX (dir.), Argumentation, manipulation, persuasion, L'Harmattan, Paris, 2007, P. 14.

${ }^{12}$. Ibid., P. 15.

${ }^{13}$. PERELMAN, Chaim, OLBRECHTS-TYTECA, Lycie, Traité de l'argumentation : la nouvelle rhétorique, Bruxelles, Editions de l'Université de Bruxelles, 1988, p. 25. 


\section{Le conflit entre l'ethos préalable et l'ethos discursif dans le discours politique de Sadate prononcé à la Knesset israélienne}

${ }^{14}$. DECLERCQUE, Gilles, L'art d'argumenter : structures rhétoriques et littéraires, Paris, Editions Universitaires, 1993, P. 38.

${ }^{15}$. AMOSSY, Ruth, L'argumentation dans le discours, Paris, Armand Colin, 2 édition, 2006, P. 57.

${ }^{16}$. Pour faciliter la lecture de l'article, nous reproduisons la traduction faite par nous-même au sein du texte, et les extraits originaux sont mis dans un annexe à la fin de l'article.

17. C'est ce que nous allons voir avec plus de détails plus loin.

${ }^{18}$. Gilles Gautier, «L'argumentation périphérique dans la communication politique : le cas de l'argument ad hominem », HERMES, nº.16, 1995, P. 170.

${ }^{19}$. AMOSSY, Ruth, L'argumentation dans le discours, Op. cit., P. 79-80.

${ }^{20}$. CHARAUDEAU, Patrick, « De l'argumentation entre les visées d'influence de la situation de communication », Op. cit., P. 29.

${ }^{21}$. C'est nous qui le soulignons.

22. AMOSSY, Ruth, « L'ethos et ses doubles contemporains. Perspectives disciplinaires », Langage et société, 3/149, 2014, P. 14.

23. CHARAUDEAU, Patrick, « De l'argumentation entre les visées d'influence de la situation de communication », Op. cit., P. 20.

${ }^{24}$. ROBRIEUX, Jean Jacques, Rhétorique et argumentation, Paris, Armand Colin, $2^{\mathrm{e}}$ édition, 2005, P. 200.

${ }^{25}$. DUCROT, Oswald, Le dire et le dit, Paris, Les Editions de Minuit, 1984, P. 201.

${ }^{26}$. MAINGUENEAU, Dominique, L'Analyse du discours, Paris, Hachette Littérature, coll. Linguistique, 1997, P. 138.

${ }^{27}$ Citons, par exemple, le verset 61 de la sourate Al-Anfâl [Le Butin] : «Et s'ils inclinent à la paix, incline toi aussi vers celle-ci et remets ta confiance en Allah, car Lui, Il entend et sait tout, (l'Audient et l'Omniscient). »

${ }^{28}$. ABDEL-ALIM, Mohamed, /albitāb al-sadāty : taḥlìl al haqul al- $\square$ aydulūjy lil hitāb alsadāty/ [Le discours sadatien : Analyse du champ idéologique du discours sadatien], le Caire, Kitâb Al-Ahaly, 1990, P. 37.

${ }^{29}$. MAINGUENEAU, Dominique, L'Analyse du discours, Op. cit., P. 138. 
${ }^{30}$. BRETON, Philippe, L'argumentation dans la communication, Paris, Editions La Découverte, 2001, P. 66-67.

${ }^{31}$. Ibid., P. 62.

32. PERELMAN, Chaim, OLBRECHTS-TYTECA, Lycie, Op. cit., P. 99.

33. ROBRIEUX, Jean Jacques, Op. cit., P. 199

${ }^{34}$. Ibid., P. 209.

35. Ibid., P. 200.

${ }^{36}$. BRETON, Philippe, Op. cit., P. 53.

${ }^{37}$. MAYAFFRE, Damon, « Dire son identité politique, Etude du discours politique français au XXe siècle », Cahiers de la Méditerranée, L'autre et l'image de soi, $\mathrm{N}^{\circ}$. 66, 2003, $\mathrm{P}$. 247.

${ }^{38}$. WOLTON, Dominique, «La communication politique : construction d'un modèle », HERMES, Le nouvel espace public, No. 4, Paris, Editions du CNRS, 1989, P. 30.

\section{Références}

Abdel-Alim, Mohamed, (1990), /alb̧itāb al-sadāty: taḥlīl alḥaql al- $\square$ aydulūjy lilbuitāb alsadāty/ [Le discours sadatien : Analyse du champ idéologique du discours sadatien], Kitâb Al-Ahaly, le Caire.

Adam, Jean-Michel, (2008), La linguistique textuelle, Armand Colin, Paris.

Seignour, Amélie, (2011), « Méthode d'analyse des discours, L'exemple de l'allocution d'un dirigeant d'entreprise publique », Revue française de gestion - Nº 211, P.29-45.

Amossy, Ruth, (2014), " L'ethos et ses doubles contemporains. Perspectives disciplinaires », in Langage et société, V.3, n 149, p. 13-30.

Amossy, Ruth, (2006), L'argumentation dans le discours, Paris, Armand Colin, 2 édition.

Amossy, Ruth, Roselyne Koren, (2010), «Argumentation et discours politique », Mots. Les langages du politique, $\mathrm{N}^{\circ} 94$ novembre 2010, Trente ans d'étude des langages du politique (1980-2010), p. 13-21.

Bonnet, Valérie, (2007), «Identité collective et ethos de l'orateur dans la chanson afroaméricaine », dans Christian BOIX (dir.) Argumentation, manipulation, persuasion, L'Harmattan, Paris, pp.249-274. 


\section{Le conflit entre l'ethos préalable et l'ethos discursif dans le discours politique de Sadate prononcé à la Knesset israélienne}

Boutin, Christophe, (2009), Les grands discours $d u X X^{e}$ siècle, Paris, Flammarion.

Breton, Philippe, (2001), L'argumentation dans la communication, Paris, Editions La Découverte, 2001.

Charaudeau, Patrick, (2007), « De l'argumentation entre les visées d'influence de la situation de communication », Christian BOIX (dir.) Argumentation, manipulation, persuasion, L'Harmattan, Paris, pp.13-35.

Charaudeau, Patrick, MAINGUENEAU, Dominique, (dirs.), (2002), Dictionnaire d'analyse du discours, Paris, Seuil.

Charaudeau, Patrick, (2008), «L'argumentation dans une problématique de l'influence », Argumentation et Analyse du Discours (AAD), no.1, (En ligne),.

Charaudeau, Patrick, (2005), Le discours politique, les masques du pouvoir, Paris, Librairie Vuibert.

Declercque, Gilles, (1993), L'art d'argumenter : structures rhétoriques et littéraires, Paris, Editions Universitaires.

Ducrot, Oswald, (1984), Le dire et le dit, Paris, Les Editions de Minuit, 1984.

Gautier, Gilles, (1995), «L'argumentation périphérique dans la communication politique : le cas de l'argument ad hominem », HERMES, no.16, p.167-185.

Haddad, Galit, (1999), «Ethos préalable et ethos discursif : l'exemple de Romain Rolland, in Ruth AMOSSY (Dir.), Images de soi dans le discours, la construction de l'ethos, Paris, Delachaux et Niestlé.

Herman, Thierry, (2005), «L'analyse de l'ethos oratoire », in Philippe LANE (dir.), Des discours aux textes: modèles et analyses, Publications des universités de Rouen et du Havre, pp.157-182.

Kerbrat-Orecchioni C., (1980), L'énonciation de la subjectivité dans le langage, Paris, Colin, 1980

Maingueneau, Dominique, (1997), L'Analyse du discours, Paris, Hachette Littérature, coll. Linguistique.

Mayaffre, Damon, (2003), «Dire son identité politique, Etude du discours politique français au XXe siècle », dans Cahiers de la Méditerranée, No. 66, L'autre et l'image de soi, p. 247264.

Mohy El-Din, Khaled, (1992), /Wal $\square \bar{a} n \square$ atakalam/ [Et maintenant je parle], le Caire, le Centre d'Al-Ahram de traduction et de publication. 
Montefiore, S. S., (2010), Ces grands discours qui ont changé le monde, de Jésus à Obama, traduit de l'anglais par Dominique Piolet-Françoise, Paris, Dunod.

Perelman, Chaim, Olbrechts-Tyteca, Lycie, (1988), Traité de l'argumentation : la nouvelle rhétorique, Bruxelles, Editions de l'Université de Bruxelles.

Robrieux, Jean Jacques, (2005), Rhétorique et argumentation, Paris, Armand Colin, 2e édition.

Wolton, Dominique, (1989), «La communication politique : construction d'un modèle », HERMES, No. 4, Le nouvel espace public, Paris, Editions du CNRS, p.27-42.

\section{Annexe : Les extraits originaux:}

$$
\begin{aligned}
& \text { 1- أنتم تريدون العيش معنا في هذه المنطقة من العالم. و أنا أقول لكم، بكل الإخلاص، إننا نرحب بكم بيننا، بكل }
\end{aligned}
$$

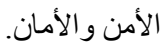

$$
\begin{aligned}
& \text { r- ولقد أعلنت أكثر من مرة، أن إسر ائيل أصبحت حقيقة واقعة. } \\
& \text { r- ولكنني أقول لكم اليوم، وأعلن للعالم كله، إننا نقبل بالعيش معكم في سلام دائم و عادل. } \\
& \text { ع - وقد جئت إليكم اليوم علي قدمين ثابتتين ، لكي نبني حياة جديدة، لكي نقيم السلام. } \\
& \text { هـ ـ لم أنداول هذا القرار مع أحد من زملائي وإخوتي، رؤساء الدول العربية، أو دول المواجهة. ولقد اعترض من بن } \\
& \text { اتصل بي منهم، بعد إعلان القرار. } \\
& \text { T- لقد جئت إليكم لكي نبني معًا السلام الدائم، العادل، حتى لا ثُراق نقطة دم واحدة من جسد عربي أو إسر ائيلي. } \\
& \text { V- ـ و هؤلاء الذين يتحملون، مثلنا، تلك المسؤولية الملقاة على عاتقنا، هم أول من يجب أن تتو افر لديهم الثجاعة }
\end{aligned}
$$

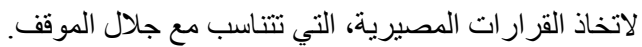

$$
\begin{aligned}
& \text { ᄉـ ولقد تحملت و أتحمل متطلبات المسؤولية التاريخية. }
\end{aligned}
$$

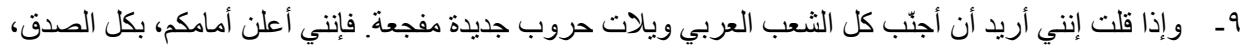

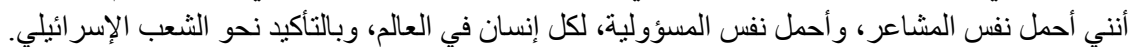

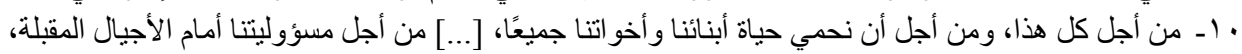

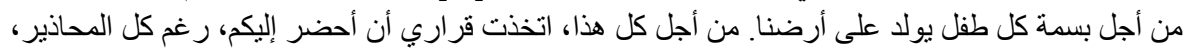

$$
\begin{aligned}
& \text { لكي أقول كلمتي. }
\end{aligned}
$$

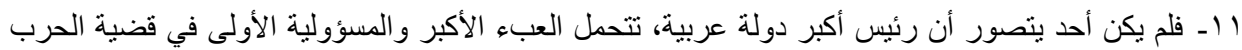

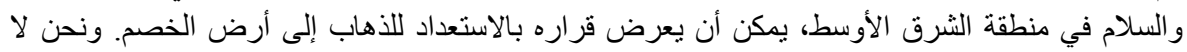

$$
\begin{aligned}
& \text { نز ال في حالة حرب. }
\end{aligned}
$$




\section{Le conflit entre l'ethos préalable et l'ethos discursif dans le discours politique de Sadate prononcé à la Knesset israélienne}

r ا ـ اصارحمم القول بكل الصدق... أعلن امامكم بكل الصدق... أرجو أيضًا أن أحذركم بكل الصدق...

با اـ و إنني ألتمس العذر لكل من استقبل قراري، عندما أعلنته للعالم كله أمام مجلس الثعب المصري، بالدهشة، بل

ـ ا ـ ـ الإنسان الذي خلقه الله، كما يقول غاندي، قدّيس السلام، "لكي يسعى على قَدَيه، يبني الحياة، ويعبد الله".

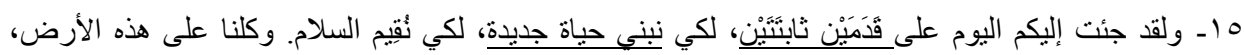

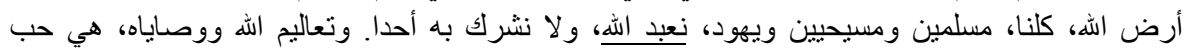

$$
\text { وصدق وطهارة وسلام. }
$$

17 ـ لماذا لا نؤمن بحكمة الخالق، التي أوردها في أمثال سليمان الحكيم: " الغش في قلب الذين يفكرون في الثرّ.

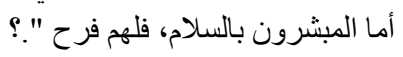

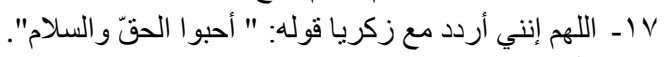

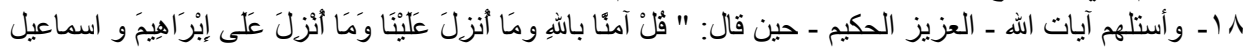

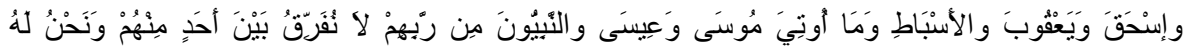

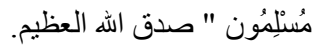

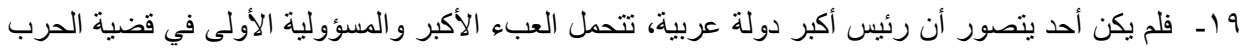

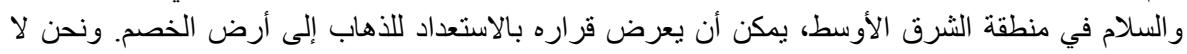

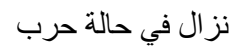

$$
\begin{aligned}
& \text { • †- لقد كيّا نرفضكم، وكانت لنا أسبابنا ودعو انا... نعم. } \\
& \text { لقد كنّا نرفض الاجتماع بكم، في أي مكان.. نعم. } \\
& \text { لقد كتّا نصفكم بإسر ائيل المز عومة.. }
\end{aligned}
$$

ا ــ لقد كان بيننا وبينكم جدار ضخم مرتفع، حاولتم أن تبنُوه على مدى ربع قرن من الزمان. ولكنه تحطم في عام

$$
\text { كان جدارًا من الحرب النفسية، المستمرة في التهابها وتصاعدها. }
$$

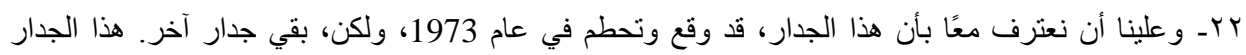

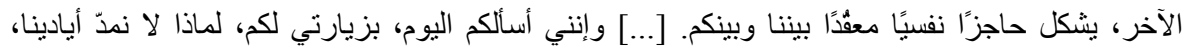

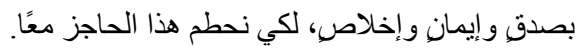

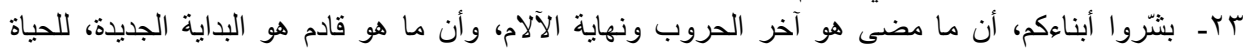

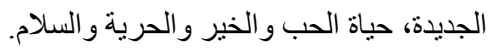

$$
\text { ويا ويا ويا أيتها الأم الثكلى، وليها الابن الزوجة المترملة، }
$$


ـ ـ املئوا الأرض و الفضاء بتر اتيل السلام....

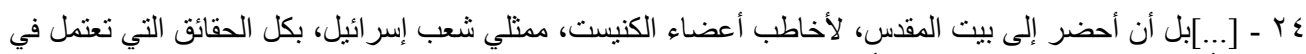

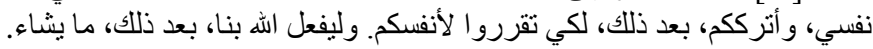

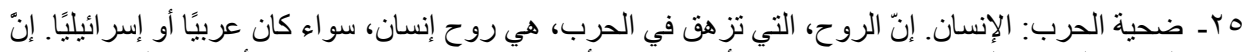

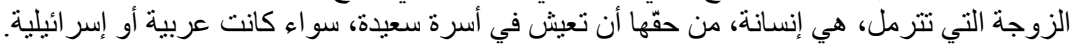

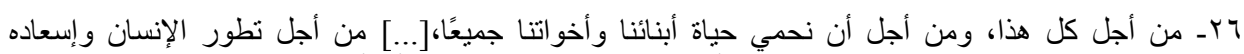

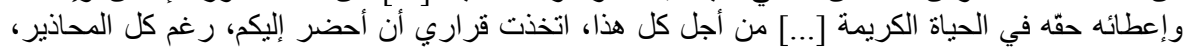
لكي أقول كلمني.

V Vـ لماذا نُورث هذه الأجيال نتائج سفلك الدماء، وإزهاق الأرواح، وتيتيم الأطفال، وترمل الزوجات، وهدم الأسر ، و أنين الضحايا؟

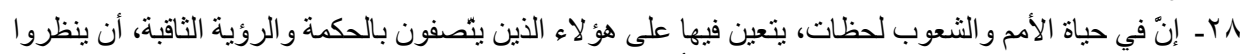

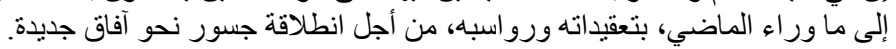

$$
\text { 9 9 - لا سعادة لأحد علي حساب شقاء الاخرين. }
$$

• بـ و لا حل لمشكلة أبدا بالهروب منها أو بالتعالي عليها. 